\title{
Multicast Transport Protocol Analysis: Self-Similar Sources*
}

\author{
Mine Çağlar' and Öznur Özkasap \\ ${ }^{1}$ Koç University, Department of Mathematics, Istanbul, Turkey \\ ${ }^{2}$ Koç University, Department of Computer Engineering, Istanbul, Turkey \\ \{mcaglar, oozkasap\} @ku. edu.tr
}

\begin{abstract}
We study the traffic that scalable multicast protocols generate in terms of message delays over the network as well as traffic counts at the link level in the case of self-similar sources. In particular, we study Bimodal Multicast and Scalable Reliable Multicast protocols proposed for scalable reliable multicasting. These protocols are based on different mechanisms for recovering from message losses and providing scalability. We discuss the protocol mechanisms as the main underlying factor in our empirical results. Our results can be considered as a contribution to the general problem of integration of multicast communication to large scale.
\end{abstract}

Keywords: Scalable multicast; loss recovery; self-similarity; long-range dependence.

\section{Introduction}

Analyses of fine-grained measurements over the last decade reveal that long-range dependence exists in the link level network traffic as well as self-similarity [1]. Selfsimilarity in the presence of long-range dependence has adverse consequences on network performance. We focus on the traffic that scalable multicast protocols generate. These protocols have been compared with respect to several performance measures such as scalability, reliability and congestion control. However, the nature of the traffic stream generated by each type of protocol particularly with respect to self-similarity has not been studied extensively.

We consider multicast communication traffic in the case of self-similar sources. In particular, we study Bimodal Multicast [2] and Scalable Reliable Multicast (SRM) [3] protocols proposed for scalable reliable multicasting. These protocols are based on different mechanisms for recovering message losses and providing scalability, namely epidemic dissemination for Bimodal Multicast and nonhierarchical feedback control for SRM. Our previous studies demonstrate that epidemic approach of Bimodal Multicast generates a more desirable traffic than SRM with lower overhead traffic and transport delays in the case of a constant bit rate source [4,5]. In this study, we consider a self-similar source, namely an on/off sender that transmits with Pareto on

This work is supported by TUBITAK and COST Action 279 "Analysis and Design of Advanced Multiservice Networks supporting Mobility, Multimedia, and Internetworking". 
and off times as an extension of [6]. The delays and traffic at the link level are studied and compared with constant bit rate case. Bimodal Multicast generates shorter delays; however long-range dependence arises in the link level. For SRM, the traffic becomes worse in terms of both delays and at the link level.

We elaborate on the protocol mechanisms as the main underlying factor in our empirical results. The intrinsic relation of these mechanisms to traffic characteristics is explored. Our results can be considered toward the general problem of integration of multicast communication to the Internet. The ultimate aim is to discover and develop multicast protocols that not only feed well-behaved traffic discretely into the existing networks, but also can cope with the existing self-similar traffic and its adverse consequences. Next section describes comparative simulation results for protocols that we investigate. Then, we give our conclusions and point at future directions.

\section{Simulations, Analysis, and Results}

The implementation of Bimodal Multicast that we developed over ns-2 [2] and the available ns-2 model of SRM are used [7]. The simulation scenario is transit-stub topology with $60,80,100$ and 120 nodes where every node is a group member. Transit-stub topologies approximate the structure of the Internet that can be viewed as a collection of interconnected routing domains where each domain can be classified as either a stub or a transit domain [8]. The sender is located on a central node and the receiver that we analyze for transport delays is located as far as possible from the sender. We also monitor a central link that is heavily loaded. A $1 \%$ drop rate set on every link forms a system-wide noise. We obtain our results from a sequence of 35000 (approximately $2^{15}$ ) multicast data messages each with size 210 bytes. We have a single on/off source which multicasts to all receivers continuously. On and off times follow a Pareto distribution with shape parameter 1.5 and mean 500 milliseconds each. On the average, 50 messages per second are transmitted like the cbr simulations of [5]. In order to estimate the Hurst parameter $H$ from the delay of these messages or from traffic counts at the link level, we apply the wavelet estimation method as given in [9] using Daubechies wavelets with three vanishing moments.

Bimodal Multicast [2] which is based on an epidemic loss recovery mechanism is a novel option in the spectrum of multicast protocols. It has been shown to impose constant loads on links and routers if configured correctly and to exhibit stable throughput under failure scenarios that are common on real large-scale networks. In contrast, this kind of behavior can cause other reliable multicast protocols to yield unstable throughput. Bimodal Multicast consists of two sub-protocols, namely an optimistic dissemination protocol and a two-phase anti-entropy protocol. The former is a best-effort, hierarchical multicast used to efficiently deliver a multicast message to its destinations. This phase is unreliable and does not attempt to recover a possible message loss. When available, IP multicast, or a randomized dissemination protocol can be used. The second stage is responsible for message loss recovery. It is based on an anti-entropy protocol that detects and corrects inconsistencies in a system by continuous gossiping. We refer the interested reader to [2] for further details on Bimodal Multicast and the theory behind the protocol. 


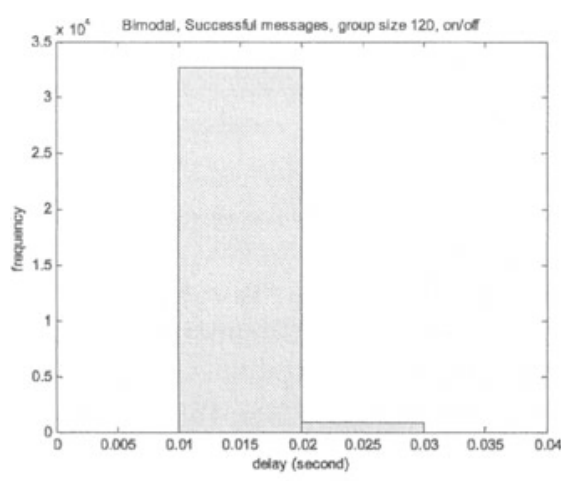

(a)

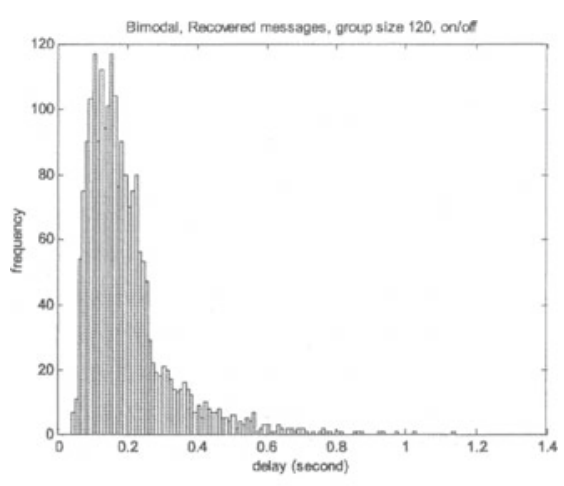

(b)

Fig. 1. Marginal delay distribution of (a) successful (b) recovered messages with Bimodal Multicast for group size 120.

In view of the simulation results in [5], Bimodal Multicast has an exponential marginal delay distribution and a Markovian recovery mechanism. As a result, LRD is not an expected phenomenon. Indeed, the delay sequence obtained with a cbr source shows no LRD in the case of Bimodal Multicast whereas the delay of SRM is long range dependent for larger group sizes 100 and 120 [5]. The transport layer mainly translates LRD from application level to link level while recent studies show that it can also cause LRD and self-similarity up to the scale of minutes, not only at small scales. This has been shown for TCP, which is the prevalent protocol over the Internet. This leads us to the question: what happens if the source does not multicast with cbr but mimics an on/off source, which is typical in current networks? It is well known that when sufficiently many of traffic streams from such sources are aggregated, LRD arises at the link level [10].

The marginal delay distributions for both Bimodal Multicast and SRM do not change in general with an on/off source. Figures 1 and 2 show these distributions separately for messages that are successful in the initial multicast and for those recovered through loss recovery mechanism. Bimodal Multicast has an exponential delay distribution as given in Fig. 1 (b). For the group size 120, LRD has been detected for SRM with a cbr source where the successful messages follow a normal distribution [5]. In the on/off case, this distribution becomes lognormal with a pronounced right tail as shown in Fig. 2 (a). The mean delays are slightly higher in SRM for group sizes 100 and 120 with an on/off source, but not significantly. The means are plotted for all group sizes in Fig. 3 comparatively with cbr results. As in the cbr case, SRM delay is much higher than Bimodal delay.

The correlations in delay are measured through the Hurst parameter $H$. If the delay sequence is self-similar with LRD, then $H$ takes values in $(0.5,1)$. It has been found that neither the delay sequence nor the link level traffic of Bimodal Multicast show LRD. However SRM shows LRD for larger group sizes even with a cbr source. The TCP-like approach of SRM induces LRD and self-similarity. SRM necessitates the basic IP delivery model and forms reliability on an end-to-end basis. Similar to TCP that adaptively sets timers or congestion control windows, SRM algorithms 


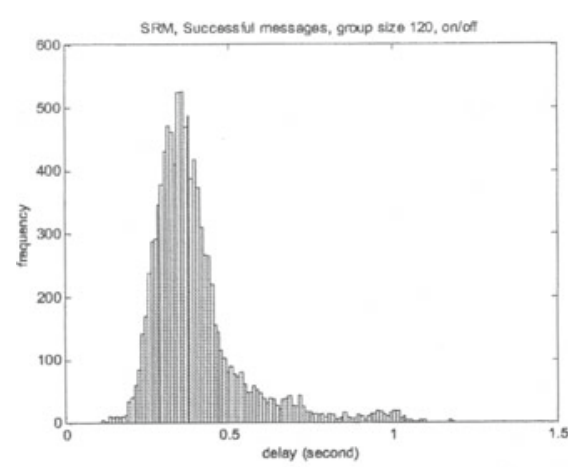

(a)

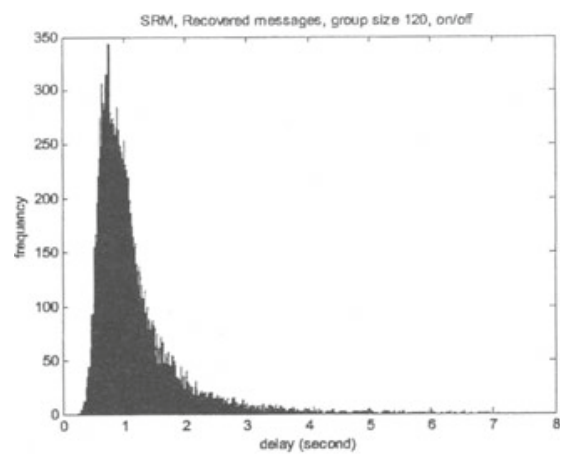

(b)

Fig. 2. Marginal delay distribution of (a) successful (b) recovered messages with SRM for group size 120 .

dynamically regulate their control parameters such as request and repair timers, based on the observed performance within a session. Repair requests and retransmissions are multicast to the whole group. A lost packet ideally triggers only a single request from a host just downstream of the point of failure.

With an on/off source, the Hurst parameter estimates are given in Fig. 4 where $H$ is simply estimated as 1 in group sizes 100 and 120 for SRM. In fact, the scaling diagrams in these sizes do not show a linear scaling even for larger scales and indicate a multifractal sequence. Therefore, a linear fit yields $H$ greater than 1 . The same value is obtained for the delay of recovered messages. This was not the case with a cbr source; we had estimated $H$ to be around 0.8 for both group sizes with a clearly selfsimilar delay sequence. On the other hand, Bimodal Multicast delays are scalable with respect to group size as $H$ remains around 0.5 all throughout.

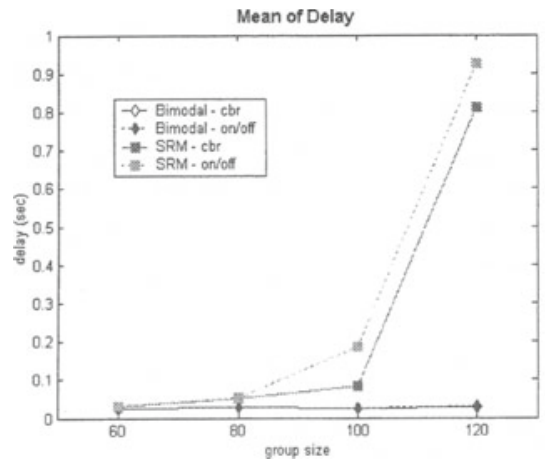

Fig. 3. Mean delay of a message

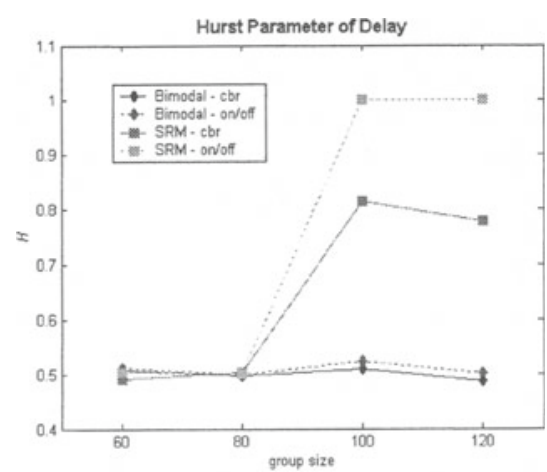

Fig. 4. Hurst parameter obtained from delay sequence

We measure goodput as the ratio of the total number of messages successfully received to the total number received including protocol overhead. Fig. 5 shows that goodput remains relatively constant in Bimodal Multicast whereas it decreases 


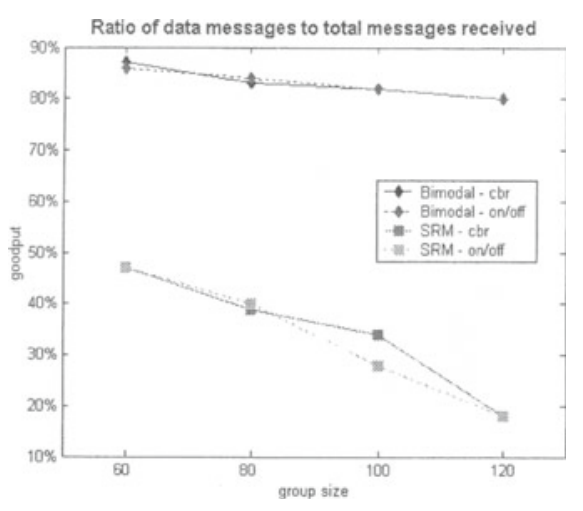

Fig. 5. Goodput versus group size

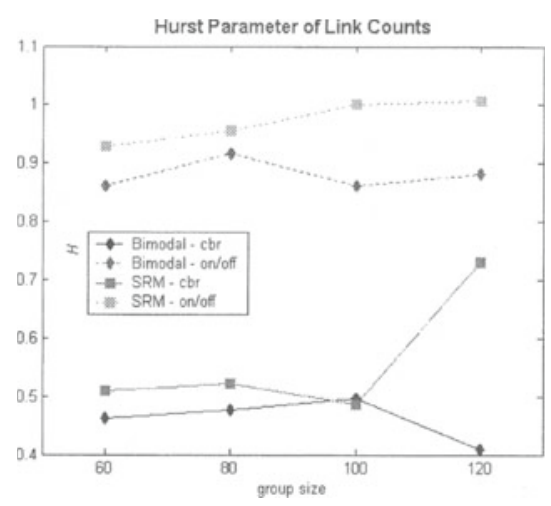

Fig. 6. Hurst parameter obtained from link counts

significantly in SRM as the group size increases. These results are very close to those with a cbr source [5]. In view of Hurst parameter calculations, we can conclude that the correlations for several performance indicators worsen with an on/off source whereas the means do not get much affected.

In comparison to the transport level, the link level results are different for Bimodal Multicast but similar for SRM in terms of LRD. Fig. 6 gives the Hurst parameters estimated from a monitored link incoming to our fixed receiver. The results from the reverse direction are similar with slightly lower $H$ values and significantly lower mean. As the receiver is chosen to be one of the end nodes, the latter direction is less loaded. Bimodal Multicast shows LRD at the link level with an on/off source. In fact, this is not surprising. Although there is a single on/off source, at the link level there is an aggregation arising from the recovery process of all receivers in the network. If the traffic streamed to a few tens of these members multiplex in this link, then the limiting self-similar behavior can be easily observed. This is due to the on/off behavior of the source being propagated by each stream. This is interesting as it indicates a slightly different aggregation of on/off sources by multicasting, rather than many sources being present in a unicast environment [10]. The scaling diagrams of the delay sequence and the link level packet counts which yield the $H$ estimates are similar in SRM. This can be explained due to self-similarity being induced by the protocol itself in addition to an on/off source.

The most important effect of an on/off source on Bimodal Multicast traffic is the emergence of LRD at the link level. Another observation has been an increase in message loss. There is a significant probability that there will be high delays for some messages with a Pareto on/off source although these messages are few. Therefore, the mean delay does not increase significantly but we can detect more message loss. The number of rounds for holding a lost message in the buffer is a parameter of the Bimodal Multicast simulation and is called the stability threshold. By default, this parameter is set to 10 . We have observed that increasing the stability threshold decreases loss. 
We have already shown that SRM induces self-similarity on both delays at the transport level and the traffic counts at the link level even in the case of a cbr source. The immediately observed effect of an on/off source is that LRD gets more pronounced. In fact, a monofractal model of self-similarity does not hold in this case. In the scaling diagram of the delay sequence in the case of a cbr source, the linear scaling over larger scales is evident [5]. However, for an on/off source, the scaling is much different indicating a multifractal character. The analysis of the coupling of an on/off source with SRM's loss recovery mechanism to yield such results remains as future work.

\section{Conclusions and Future Directions}

In this study, we focus on the traffic that scalable multicast protocols generate. We consider a self-similar source, namely an on/off sender that transmits with Pareto on and off times. The delays and traffic at the link level are studied and compared with constant bit rate case. Bimodal Multicast generates desirable delays; however longrange dependence arises in the link level. For SRM, the traffic becomes worse in terms of both delays and at the link level. Our results can be considered toward the general problem of integration of multicast communication to the Internet. We have demonstrated that Bimodal Multicast is a good candidate for both generating wellbehaved traffic and also coping with the existing self-similar traffic.

\section{References}

1. Leland, W. E., Taqqu, M. S., Willinger, W. and Wilson, D. V.: On the Self-Similar Nature of Ethernet Traffic (Extended Version), IEEE/ACM Trans. On Networking, 2 (1994) 1-15

2. Birman, K.P., Hayden, M., Ozkasap, O., Xiao, Z., Budiu, M. and Minsky, Y.: Bimodal Multicast, ACM Transactions on Computer Systems, 17 (1999) 41-88

3. Floyd, S., Jacobson, V., Liu, C., McCanne, S. and Zhang, L.: A Reliable Multicast Framework for Light-weight Sessions and Application Level Framing, IEEE/ACM Transactions on Networking 5 (1997) 784-803

4. Özkasap, Ö, Çağlar, M.: Traffic Behavior of Scalable Multicast: Self-similarity and Protocol Dependence. Proceedings, International Teletraffic Congress 18, Berlin, Germany, 31 Aug.- 5 Sep. (2003)

5. Çağlar, M., Özkasap, O.: Traffic Properties of Scalable Multicast Communication: Comparison of Bimodal Multicast and SRM. Submitted (2002)

6. Özkasap, Ö, Çağlar, M.: Traffic Characterization of Scalable Multicasting in the case of a Self-Similar Source (poster), ACM SIGCOMM, Karlsruhe, Germany, 25-29 Aug. (2003)

7. Bajaj, S., Breslau, L., Estrin, D., et al.: Improving Simulation for Network Research, USC Computer Science Dept. Technical Report 99-702 (1999)

8. Calvert, K., Doar, M., and Zegura, E.W.: Modeling Internet Topology. IEEE Communications Magazine, June (1997)

9. Veitch, D. and Abry, P.: A Wavelet Based Joint Estimator of the Parameters of LongRange Dependence, IEEE Trans. on Information Theory, 45 (1999) 878-897

10. Taqqu, M.S., Willinger, W. and Sherman, R.: Proof of a Fundamental Result in SelfSimilar Traffic Modeling, Computer Communication Review, 27 (1997) 5-23 